\title{
Molecular profile of 5-fluorouracil pathway genes in colorectal carcinoma
}

\author{
T. Kunicka ${ }^{1,2,3}$, P. Prochazka ${ }^{4}$, I. Krus ${ }^{1}$, P. Bendova ${ }^{3,4}$, M. Protivova' ${ }^{1}$, S. Susova ${ }^{1,3}$, V. Hlavac ${ }^{1,2,3}$, V. Liska ${ }^{3,5}$, P. Novak, \\ M. Schneiderova ${ }^{6}$, P. Pitule ${ }^{3}$, J. Bruha ${ }^{3,5}$, O. Vycital ${ }^{3,5}$, P. Vodicka ${ }^{3,4^{*}}$ and P. Soucek ${ }^{1,3,7^{*}}$
}

\begin{abstract}
Background: This study addresses involvement of major 5-fluorouracil (5-FU) pathway genes in the prognosis of colorectal carcinoma patients.

Methods: Testing set and two validation sets comprising paired tumor and adjacent mucosa tissue samples from 151 patients were used for transcript profiling of 15 5-FU pathway genes by quantitative real-time PCR and DNA methylation profiling by high resolution melting analysis. Intratumoral molecular profiles were correlated with clinical data of patients. Protein levels of two most relevant candidate markers were assessed by immunoblotting.

Results: Downregulation of DPYD and upregulation of PPAT, UMPS, RRM2, and SLC29A1 transcripts were found in tumors compared to adjacent mucosa in testing and validation sets of patients. Low RRM2 transcript level significantly associated with poor response to the first-line palliative 5-FU-based chemotherapy in the testing set and with poor disease-free interval of patients in the validation set irrespective of 5-FU treatment. UPP2 was strongly methylated while its transcript absent in both tumors and adjacent mucosa. DPYS methylation level was significantly higher in tumor tissues compared to adjacent mucosa samples. Low intratumoral level of UPB1 methylation was prognostic for poor disease-free interval of the patients $(P=0.0002)$. The rest of the studied 5-FU genes were not methylated in tumors or adjacent mucosa.

Conclusions: The observed overexpression of several 5-FU activating genes and DPYD downregulation deduce that chemotherapy naïve colorectal tumors share favorable gene expression profile for 5-FU therapy. Low RRM2 transcript and UPB1 methylation levels present separate poor prognosis factors for colorectal carcinoma patients and should be further investigated.
\end{abstract}

Keywords: Colorectal carcinoma, 5-fluorouracil, Methylation, Expression, Prognosis

\section{Background}

Colorectal carcinoma (OMIM: 114500) is the third most common malignancy and the fourth cause of cancerrelated deaths in the adult population worldwide, with the highest incidence recorded in Central Europe [1, 2].

Colorectal cancer treatment consists of surgical removal of the tumor and, based on disease characteristics, of chemo- and or radiotherapy. 5-Fluorouracil (5-FU) is widely used drug in the first-line therapy of colorectal cancer [3]. Over $80 \%$ of administered 5-FU dose is

\footnotetext{
*Correspondence: pvodicka@biomed.cas.cz; pavel.soucek@szu.cz ${ }^{3}$ Biomedical Centre, Medical School Pilsen, Charles University in Prague, Pilsen, Czech Republic

'Department of Toxicogenomics, National Institute of Public Health, Prague, Czech Republic

Full list of author information is available at the end of the article
}

rapidly degraded [4] and only $1-3 \%$ is converted into its active metabolite fluorodeoxyuridine monophosphate (FdUMP [5],). FdUMP then inhibits thymidylate synthase (TYMS, OMIM: 188350) and blocks deoxythymidine triphosphate (dTTP) synthesis. Subsequent dTTP depletion triggers "thymineless" death [6]. TYMS is considered as a potential prognostic marker for colorectal cancer. Recent studies have shown that overexpression of TYMS transcript predicts poor outcome in colorectal cancer patients $[7,8]$. However, another contemporary study has not confirmed these observations as intratumoral TYMS transcript level was not predictive in patients with colorectal cancer of stage II and III [9].

Several studies have indicated potential prognostic or predictive role of 5-FU metabolizing enzymes expression 
for resistance to the treatment of colorectal cancer. Colorectal cancer patients with low protein expression of 5-FU inactivating enzyme dihydropyrimidine dehydrogenase (DPYD, OMIM: 612778) exhibited a longer survival after 5-FU-treatment than those with high levels [10]. Likewise, high DPYD transcript level was associated with poor outcome of stage IV colorectal cancer patients [11]. High thymidine phosphorylase (TYMP, OMIM: 131222, 5-FU activating enzyme) transcript level was associated with significantly better disease-free survival (DFS) following oral administration of 5-FU in stage III colorectal cancer patients [12].

The resistance of the tumor cells towards $5-\mathrm{FU}$ is substantially modulated by the transport mechanisms. Especially solute carrier transporter 29A1 (SLC29A1, OMIM: 602193) plays a crucial role in cellular uptake of nucleoside drugs such as cytarabine, gemcitabine, or 5-FU [13]. Results of a recent small scale functional study suggested that high SLC29A1 mRNA levels in colorectal cancer tumor tissue correlate with poor clinical response to 5-FU [14].

In this study we aimed to address importance of gene expression and methylation profile of 155 -FU genes in tumor and adjacent bowel mucosa tissues of colorectal cancer patients for the patient's prognosis and the response to 5 -FU. Genes were selected from literature and PharmGKB database based on functional evidence from 5FU pharmacokinetics data (https://www.pharmgkb.org/). Protein expression of two most relevant candidate markers was assessed as another chain underlying 5-FU mode of action.

\section{Methods}

\section{Studied patients and collection of biological specimen}

Tumor tissue and adjacent non-neoplastic mucosa samples were obtained from total of 151 patients with sporadic colorectal cancer (C18-21 according to ICD-10) diagnosed at the Department of Surgery and Oncology, Teaching Hospital and Medical School in Pilsen, and General Teaching Hospital in Prague between January 2008 and November 2011. From 151 patients, 146 paired tissue samples (tumor and control mucosa), four tumors, and one mucosa sample were taken for analyses (for study flow chart, see Fig. 1). Native tissue samples were collected as described elsewhere $[15,16]$.

Patients represented three groups - testing set (stage II-IV, $n=52$ ) for gene and protein expression and methylation analysis, validation set I (stage II, $n=67$ ) for gene expression analysis, and validation set II (stage II and III, $n=32$ ) for gene expression and methylation analysis. The lack of tissue aliquots for simultaneous isolation of RNA and DNA necessitated the use of two different validation sets. All patients in the testing set underwent adjuvant $(n=26)$ or palliative $(n=26)$ chemotherapy regimens based on 5-FU (with added leucovorin and/or oxaliplatin). In the validation sets I and II, 24 and 17 patients were treated by such chemotherapy regimens, respectively (Table 1 and Fig. 1).

Response to the palliative treatment was evaluated by RECIST criteria [17] based on routine imaging techniques for assessment of tumor mass (computerized tomography with or without positron emission, magnetic resonance or ultrasonography). Increase in tumor mass or the appearance of new lesions in patients with palliative treatment indicated progression and thus poor response to the treatment (PD). Good response to the treatment was defined as a decrease of the number or volume of metastases, i.e., complete or partial response (CR or PR) or stabilization of the disease or (SD). In patients treated by adjuvant therapy after radical surgical resection R0 disease-free interval (DFI) served as a measure of the treatment outcome. DFI was defined as the time elapsed between radical surgical R0 resection and disease recurrence.

Methylation analyses were conducted on 22 tissue pairs from the testing set and on the whole independent validation set II from the General Teaching Hospital, Prague.

\section{Isolation of total RNA and CDNA synthesis}

Total RNA was isolated from frozen tissues using Trizol $^{\circ}$ reagent (Life Technologies, Carslbad, CA), stored, and characterized for the quantity and quality [18]. Complementary DNA (cDNA) was synthesized using $0.5 \mu \mathrm{g}$ of total RNA and random hexamer primers with help of RevertAid $^{\text {Tw }}$ First Strand cDNA Synthesis Kit (MBI Fermentas, Vilnius, Lithuania). Quality of cDNA in terms of DNA contamination was confirmed by PCR amplification of ubiquitin $C$ [19].

\section{Gene expression profiling}

Quantitative real-time PCR (qPCR) was performed using ViiA7 Real-Time PCR System, TaqMan ${ }^{\circ}$ Gene Expression Assays and TaqMan ${ }^{\circ}$ Gene Expression Master Mix (Life Technologies). Reference genes - POLR2A (DNAdirected RNA polymerase II subunit A, OMIM: 180660), MRPL19 (mitochondrial ribosomal protein L19, OMIM: 611832), EIF2B1 (eukaryotic translation initiation factor 2B, subunit 1, OMIM: 606686), and PSMC4 (proteasome 26S subunit, ATPase, 4, OMIM: 602707) - were selected by us earlier [15]. Gene Expression Assays with their characteristics are listed in Additional file 1: Table S1. While samples from the testing set were preamplified using TaqMan PreAmp Master Mix (Life Technologies), cDNA from the validation sets was used for quantification directly without preamplification procedure [20]. For calculating the qPCR efficiency of each assay, a calibration curve from one non-neoplastic sample was 


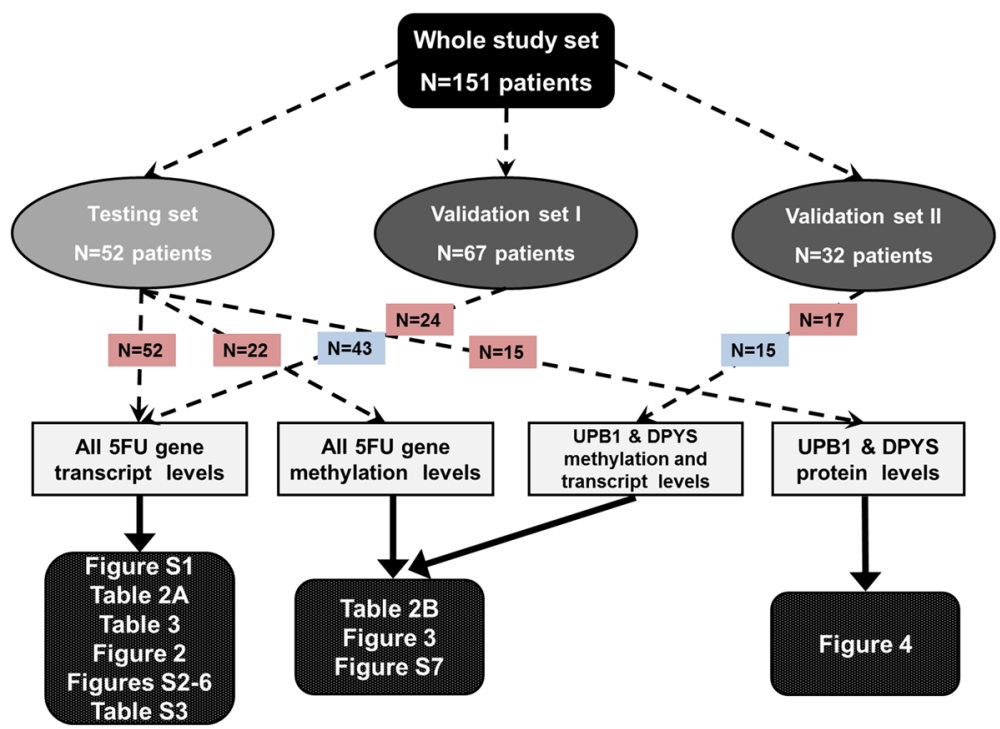

Fig. 1 Flow chart of the study. Samples flow and experimental data are displayed by dashed lines and statistical analyses by solid lines. Numbers of 5-FU treated patients in red rectangles and untreated patients in blue rectangles

prepared (six points, 5-times dilution). The non-template control contained water instead of cDNA.

The qPCR study design adhered to the MIQE Guidelines (Minimum Information for Publication of Quantitative Real-Time PCR Experiments [21]).

Gene expression and clinical data of all samples were submitted to Gene Expression Omnibus (GEO) repository under accession number GSE67111.

\section{Promoter CpG methylation profiling}

To convert unmethylated cytosines to uracils whole genomic DNA was treated with sodium bisulfite using the Epitect Bisulfite Kit (Qiagen, Hilden, Germany) following the manufacturer's protocol. Promoter region of every gene of interest was determined using Genomatix MatInspector and Genes \& Genomes software (Genomatix Software GmbH, Munich, Germany). CpG islands or simple CpG sites were identified by Methyl Primer Express Software v1.0 (Applied Biosystems, Foster City, CA). The same software was used for design of primers specific for sodium bisulfite converted DNA bases. Number of CpGs in the PCR amplicon and equal primer melting temperature ( $\mathrm{Tm}$ ) were taken into consideration in the primer design. Real-time PCR followed by high resolution melting (HRM) was carried out in highperformance Eco Real-Time PCR system (Illumina, San Diego, CA), essentially as described in [16]. PCR was initiated by incubation at $95{ }^{\circ} \mathrm{C}$ for $5 \mathrm{~min}$, followed by 50 cycles at $95{ }^{\circ} \mathrm{C}$ for $10 \mathrm{~s}$, annealing temperature of specific primers (Ta) for $20 \mathrm{~s}$, and $72{ }^{\circ} \mathrm{C}$ for $10 \mathrm{~s}$. Primer sequences, Tm, Ta, length, and numbers of CpGs for each amplicon are listed in Additional file 1: Table S2. HRM thermal profile was set up according to the manufacturer's recommendations (Qiagen). Fluorescence data were converted into melting peaks by the Eco Software (Illumina, Ver. 3.0.16.0). For each assay, a standard dilution series of EpiTect Control DNAs (Qiagen) was run to assess the quantitative properties and sensitivity of the assay. Fluorescence of each sample was normalized against $100 \%$ methylated DNA control. Methylation data of individual samples were subtracted from calibration curve with positive controls of $100,75,50,25$, and $0 \%$ methylated DNA.

\section{Immunoblotting in human colorectal cancer tissues}

Tissue sample pairs from 15 patients and unpaired tumors from two patients were selected based on tissue availability from the testing set and used for immunoblotting. Samples, stored at $-80{ }^{\circ} \mathrm{C}$ prior to the protein isolation, were grinded by a mortar and pestle, subsequently protein and total RNA were isolated using $50 \mathrm{mM}$ Tris- $\mathrm{HCl}$, $150 \mathrm{mM} \mathrm{NaCl}, 10$ \% Triton X-100 buffer. Protein concentration was determined by bicinchoninic acid assay (Thermo Scientific Pierce Protein Research Products, Rockford, IL). Immunoblotting was performed as described in [20, 22]. Briefly, $10 \mu \mathrm{g}$ of protein was used for separation by sodium dodecyl sulfate polyacrylamide gel electrophoresis (10 \%) and transferred onto $0.2 \mu \mathrm{m}$ Protran nitrocellulose membrane (Whatman, Kent, UK). Primary antibodies against dihydropyrimidinase (DPYS, OMIM: 613326) (dilution 1:4000), beta-ureidopropionase (UPB1 OMIM: 606673) (dilution 1:500) (both from Aviva System Biology, San Diego, CA), $\beta$-actin (dilution 1:2000; Sigma-Aldrich, St. Louis, MO) and the corresponding horseradish-peroxidase-conjugated secondary antibodies (dilution 1:10000; Sigma-Aldrich) were employed. Protein 
Table 1 Clinical-pathological characteristics of studied groups of patients

\begin{tabular}{llll}
\hline Characteristics & $\begin{array}{l}\text { Testing set } \\
(n=52)\end{array}$ & $\begin{array}{l}\text { Validation set I } \\
(n=67)\end{array}$ & $\begin{array}{l}\text { Validation set II } \\
(n=32)\end{array}$ \\
\hline $\begin{array}{l}\text { Gender } \\
\text { (male/female) }\end{array}$ & $36 / 16$ & $45 / 22$ & $19 / 13$ \\
Age at diagnosis $^{\mathrm{a}}$ & $63.9 \pm 9.2$ years & $70.2 \pm 9.5$ years & $70.8 \pm 11.2$ years \\
$\begin{array}{l}\text { Tumor size (pT) } \\
\text { pT2 }\end{array}$ & 3 & - & 4 \\
PT3 & 40 & 62 & 24 \\
PT4 & 9 & 5 & 4
\end{tabular}

Presence of lymph node metastasis ( $\mathrm{pN}$ )

$\begin{array}{llll}\mathrm{pN} 0 & 15 & 67 & 18 \\ \mathrm{pN1} 1-2 & 37 & - & 14\end{array}$

Presence of distant metastasis (pM)

\begin{tabular}{|c|c|c|c|}
\hline pM0 & 26 & 67 & 32 \\
\hline pM1 & 26 & - & - \\
\hline \multicolumn{4}{|l|}{ Stage } \\
\hline UICC II & 8 & 67 & 18 \\
\hline UICC III & 18 & - & 14 \\
\hline UICC IV & 26 & - & - \\
\hline \multicolumn{4}{|l|}{ Histological grade $(G)^{b}$} \\
\hline $\mathrm{Gl}$ & 6 & 9 & 6 \\
\hline Gll & 39 & 47 & 19 \\
\hline GIII & 7 & 8 & 3 \\
\hline Gx & - & 3 & 4 \\
\hline \multicolumn{4}{|l|}{ Primary localization } \\
\hline Colon & 26 & 44 & 28 \\
\hline Rectosigmoideum & 12 & 9 & 1 \\
\hline Rectum & 14 & 14 & 3 \\
\hline \multicolumn{4}{|l|}{ Chemotherapy } \\
\hline 5-FU-based & 52 & $24^{c}$ & 17 \\
\hline None & - & $33^{c}$ & 15 \\
\hline
\end{tabular}

Footnotes:

${ }^{\mathrm{a}}$ Median \pm standard deviation

${ }^{\mathrm{b}} \mathrm{G} /$ well differentiated, GII moderately differentiated, GIII poorly differentiated, Gx cannot be assessed

'Numbers may not add up to 67 of available subjects because of missing data $(n=10)$

bands were visualized with an enhanced chemiluminescence detection system (Pierce Biotechnology) by Fc Odyssey (Licor Biotechnology, Lincoln, NE) and quantified by densitometry (Image Studio software, Licor Biotechnology).

\section{Statistical analyses}

Expression levels of genes were analyzed by ViiA7 System Software (Life Technologies) and statistical analysis was performed using SPSS v16.0 Software (SPSS Inc., Chicago, IL). Fold changes were calculated usig raw cycle threshold (Ct) data by the REST2009 program (Qiagen), which is routinely used for the determination of differences between different types of sample and control groups and considers both normalization to numerous reference genes and reaction efficiencies [23]. Then ratios of $\mathrm{Ct}$ values of genes of interest and mean value of reference genes were calculated and used for further statistical analyses. Differences in gene expression or methylation levels between tumor and control tissues were assessed by the nonparametric Mann-Whitney U-test. To evaluate associations of transcript levels with clinical data and other variables (Table 1), nonparametric tests (the Kruskal-Wallis, the Mann-Whitney, and the Spearman's tests) were used.

DFI was evaluated by the Kaplan-Meier method and the Log Rank test was used for evaluation of the compared subgroups and combined groups of patients. Stage-adjusted analysis was performed by the Cox regression. All $P$-values were calculated from two-sided tests. $P$-values lower than 0.05 were considered statistically significant. The correction for multiple testing was applied according to Bonferroni.

\section{Results}

\section{Patients' characteristics}

Summary of patient's characteristics and clinical data from testing and validation sets are presented in Table 1 and the study flow diagram in Fig. 1. Testing set comprised colorectal cancer patients with stages UICC II-IV treated by first-line adjuvant $(n=26$, UICC II and III) and palliative chemotherapy based on 5-FU $(n=26$, UICC IV). Testing set served as a hypothesis generating screen and for assessment of protein levels. Validation set I used for validation of gene expression study included patients with UICC II stage $(n=67)$. Part of them was treated by 5 -FU-based chemotherapy $(n=24)$. Validation set II used for methylation study consisted of patients with UICC II and III stage $(n=32)$ with 17 patients treated by 5 -FU-based chemotherapy. The validation set II served for validation of correlations between DPYS and UPB1 methylation and expression levels and clinical data, mainly DFI. Median DFI of the validation set I was $46 \pm 6$ months and that of the validation set II was $39 \pm 3$ months.

\section{Transcript levels in tumors and non-neoplastic control tissues}

Phosphoribosylpyrophosphate amidotransferase (PPAT, OMIM: 172450), uridine monophosphate synthetase (UMPS OMIM: 613891), ribonucleotide reductase M2 (RRM2, OMIM: 180390), and SLC29A1 transcripts were consistently overexpressed in tumors compared to adjacent mucosa in both testing and validation I sets (except UMPS, all passed the correction for multiple testing, 
Table 2a, Additional file 1: Table S1). On the contrary, DPYD was downregulated in tumors compared to adjacent mucosa $(P<0.001$, both sets $)$.

\section{Associations of transcript levels with clinical data of patients}

We first tested associations between gene expression levels and therapy response of stage IV patients. Patients from the testing set with poor response to the first-line palliative treatment with 5-FU-based regimens had significantly lower expression of UMPS, ribonucleotide reductase M1 (RRM1, OMIM: 180410), and RRM2 in adjacent mucosa $(n=26 ; P=0.024, P=0.014$, and $P=0.038$, respectively; none passed the correction for multiple testing) than good responders (Table 3). Stage IV patients were excluded from subsequent survival analyses due to the metastatic character of their disease, which strongly modifies their prognosis.

For DFI analyses, transcript levels were first divided by their median separately in testing and validation set I and for the combined analysis these data were put together to eliminate raw data differences between sets. Significance of RRM2 gene expression for prognosis of colorectal cancer patients was further corroborated in the validation set I, where patients with intratumoral RRM2 transcript level higher than

Table 2 Differences in transcript (A) and methylation (B) levels between tumor and adjacent mucosa tissues of colorectal cancer patients

\begin{tabular}{|c|c|c|c|c|}
\hline \multicolumn{5}{|c|}{ A. Transcript levels } \\
\hline \multirow[t]{3}{*}{ Gene } & \multicolumn{2}{|c|}{ Testing set $(n=52)$} & \multicolumn{2}{|c|}{ Validation set I $(n=67)$} \\
\hline & Fold change & $P$-value ${ }^{\mathrm{a}}$ & Fold & $P$-value \\
\hline & \multicolumn{2}{|c|}{ Tumor vs mucosa } & \multicolumn{2}{|c|}{ Tumor $v s$ mucosa } \\
\hline DPYD & $0.45^{\mathrm{c}}$ & $<0.001$ & $0.47^{\mathrm{c}}$ & $<0.001$ \\
\hline DPYS & 0.21 & 0.014 & ND & ND \\
\hline PPAT & $2.21^{\mathrm{c}}$ & $<0.001$ & $1.95^{\mathrm{c}}$ & $<0.001$ \\
\hline RRM1 & 1.10 & 0.378 & 1.04 & 0.769 \\
\hline RRM2 & $1.68^{\mathrm{c}}$ & $<0.001$ & $1.98^{\mathrm{c}}$ & $<0.001$ \\
\hline SLC29A1 & $2.50^{\mathrm{c}}$ & $<0.001$ & $2.51^{\mathrm{c}}$ & $<0.001$ \\
\hline TK1 & 1.64 & 0.008 & 1.27 & 0.051 \\
\hline TYMP & 1.36 & 0.211 & $1.57^{\mathrm{c}}$ & 0.002 \\
\hline TYMS & 1.20 & 0.579 & 1.29 & 0.390 \\
\hline UCK1 & 0.86 & 0.126 & $0.75^{\mathrm{c}}$ & $<0.001$ \\
\hline UCK2 & 1.17 & 0.355 & 1.01 & 0.680 \\
\hline UMPS & 1.26 & 0.024 & 1.18 & 0.010 \\
\hline UPB1 & 0.71 & 0.074 & 0.65 & 0.012 \\
\hline UPP1 & 0.86 & 0.107 & $0.71^{\mathrm{c}}$ & $<0.001$ \\
\hline UPP2 & ND & ND & ND & ND \\
\hline
\end{tabular}

\section{B. Methylation levels}

\begin{tabular}{|c|c|c|c|c|c|c|}
\hline \multirow[t]{2}{*}{ Gene } & \multicolumn{2}{|c|}{ Testing set $(\mathbf{n}=\mathbf{2 2})^{d}$} & \multirow[t]{2}{*}{$P$-value } & \multicolumn{2}{|c|}{ Validation set II $(n=32)^{d}$} & \multirow[t]{2}{*}{$P$-value } \\
\hline & Tumor & Mucosa & & Tumor & Mucosa & \\
\hline$D P Y S$ & $0.19 \pm 0.14$ & $0.08 \pm 0.04$ & $<0.001^{\mathrm{c}}$ & $0.28 \pm 0.16$ & $0.20 \pm 0.13$ & $0.010^{\mathrm{c}}$ \\
\hline$U P B 1$ & $0.55 \pm 0.14$ & $0.65 \pm 0.15$ & 0.022 & $0.79 \pm 0.13$ & $0.81 \pm 0.10$ & 0.436 \\
\hline$U P P 2$ & $0.62 \pm 0.27$ & $0.56 \pm 0.25$ & 0.437 & ND & ND & ND \\
\hline
\end{tabular}

${ }^{a}$ Analyzed by the Mann-Whitney test

${ }^{b}$ Fold changes calculated by the REST2009 program

${ }^{\mathrm{c}}$ Results, which passed correction for multiple testing

${ }^{\mathrm{d}}$ Mean \pm standard deviation of percentage of sample methylation normalized to positive control (Methods)

ND not determined

Results from the testing set that have been confirmed in the validation set of patients are depicted in grey 
Table 3 Differences in transcript levels in colorectal mucosa between poor and good responders to 5-FU-based chemotherapy. Transcript levels of 5-FU pathway genes were compared in mucosas of patients in the testing set divided into groups of poor responders $(n=13)$ and good responders $(n=13)$ to the first line chemotherapy regimens based on 5-FU

\begin{tabular}{|c|c|c|c|}
\hline \multirow[t]{2}{*}{ Gene } & \multicolumn{3}{|c|}{ Expression level in poor responders vs. good responders } \\
\hline & Fold difference ${ }^{b}$ & Standard error ${ }^{\mathrm{b}}$ & $P$-value ${ }^{a}$ \\
\hline DPYD & 0.76 & $0.31-1.69$ & 0.259 \\
\hline DPYS & 0.91 & $0.10-6.89$ & 0.434 \\
\hline PPAT & 0.88 & $0.20-3.63$ & 0.086 \\
\hline RRM2 & 0.31 & $0.11-1.46$ & 0.038 \\
\hline RRM1 & 0.59 & $0.22-1.18$ & 0.014 \\
\hline SLC29A1 & 0.76 & $0.17-2.73$ & 0.369 \\
\hline TK1 & 0.87 & $0.18-3.66$ & 0.157 \\
\hline TYMP & 0.56 & $0.12-2.46$ & 0.130 \\
\hline TYMS & 0.82 & $0.15-3.77$ & 0.121 \\
\hline UCK1 & 0.85 & $0.23-1.96$ & 0.369 \\
\hline UCK2 & 0.74 & $0.17-2.42$ & 0.681 \\
\hline UMPS & 0.68 & $0.23-1.21$ & 0.024 \\
\hline UPB1 & 0.91 & $0.26-2.85$ & 0.479 \\
\hline UPP1 & 0.61 & $0.16-2.10$ & 0.106 \\
\hline
\end{tabular}

Footnotes:

${ }^{a}$ Analyzed by the Mann-Whitney test

${ }^{b}$ Fold changes and standard error calculated by the REST2009 program Significant results in bold

median had significantly longer DFI compared to patients with levels below the median $(n=66, P=0.009$, did not pass the correction for multiple testing, Fig. $2 \mathrm{a}$, the rest of results in Additional file 1: Figure S2). A non-significant association in the same direction, was observed in the testing set ( $n=26$, Additional file 1: Figure S3). Analysis of the combined testing and validation I sets supported the findings of the validation set I for RRM2 $(n=92$, $P=0.006$, did not pass the correction for multiple testing, Fig. $2 \mathrm{~b}$, the rest of results provided in Additional file 1: Figure S4). This association was significant also in stageadjusted analysis by the Cox regression of the combined set $(n=92, P=0.013, \mathrm{HR}=4.17,95 \% \mathrm{CI}=1.35-12.50$, for all results see Additional file 1: Table S3).

Then the combined set was analyzed in respect to chemotherapy by 5 -FU containing regimens $(n=50)$. However, in the combined analysis of 5-FU-treated patients from the testing and validation I sets, neither RRM2 transcript level $(P=0.301)$ nor levels of the rest of genes did significantly associate with DFI (Additional file 1: Figure S5). Stage-adjusted analysis has shown significant association between UPB1 and DFI $(P=0.047, \mathrm{HR}=0.25,95 \% \mathrm{CI}=0.06-0.98$, for all results see Additional file 1: Table S3), which was not significant in the univariate analysis $(P=0.098$, Additional file 1: Figure S5).
In DFI analyses of untreated patients $(n=32$, all stage II from the validation set 1$)$, low level of UPB1 $(P=0.026$, did not pass the correction for multiple testing) and TYMP ( $P=0.047$, did not pass the correction for multiple testing) significantly associated with worse DFI of patients (Additional file 1: Figure S6).

\section{Methylation levels in tumors and non-malignant adjacent mucosa, associations with gene expression, and clinical characteristics}

Methylation of $\mathrm{CpG}$ islands in the regulatory regions of all studied genes was initially studied in 22 pairs of tumor and adjacent mucosa (testing set) and compared with that from the independent validation set II. In the both testing and validation II sets, methylation exceeding the limit of quantitation was detected in DPYS, UPB1, and uridine phosphorylase (UPP2, GeneID: 151531) genes in both tumor and adjacent mucosa samples (Table 2b, DPYS passed the correction for multiple testing). Significantly elevated methylation level of DPYS was recorded in tumor tissues compared to adjacent mucosa in both sets (Table 2b). Methylation level of UPB1 was lower in tumors than in adjacent mucosa in the testing set, but not in the validation set II. No difference in promoter methylation was observed for UPP2 in the testing set by comparing tumors with non-malignant mucosa.

Methylation levels in promoter regions of DPYS or UPB1 did not correlate with their corresponding transcript levels either in tumors or in adjacent mucosa samples analyzed in both sets. UPP2 transcript expression was below the limit of quantification in both testing and validation II sets suggesting that this gene is completely silenced in colorectal tumors and corresponding adjacent mucosa tissues regardless clinical characteristics.

$D P Y S$ methylation level was associated with the tumor stage in the testing set $(P=0.010$, data not shown), but not in the validation set II. Therefore, this association is not further discussed. On the other hand, patients with UPB1 methylation level below the median had significantly worse DFI than those with the methylation level above the median in both sets evaluated separately (Additional file 1: Figure S7) and combined ( $n=46$, $P=0.0002$, passed the correction for multiple testing, Fig. 3). This association was significant also in the stage-adjusted analysis by Cox regression of the combined set $(n=46, P=0.004, \mathrm{HR}=9.22,95 \% \mathrm{CI}=2.04-41.57)$.

Combined analysis of UPB1 methylation in 5-FU treated patients from testing and validation II sets failed to find significant association with DFI $(n=32, P=0.653$, data not shown). For DFI analyses, patients were divided into two groups according to the median of methylation levels in tumors. Methylation levels of DPYS and UPP2 have not associated with the DFI of patients $(P>0.05)$. 

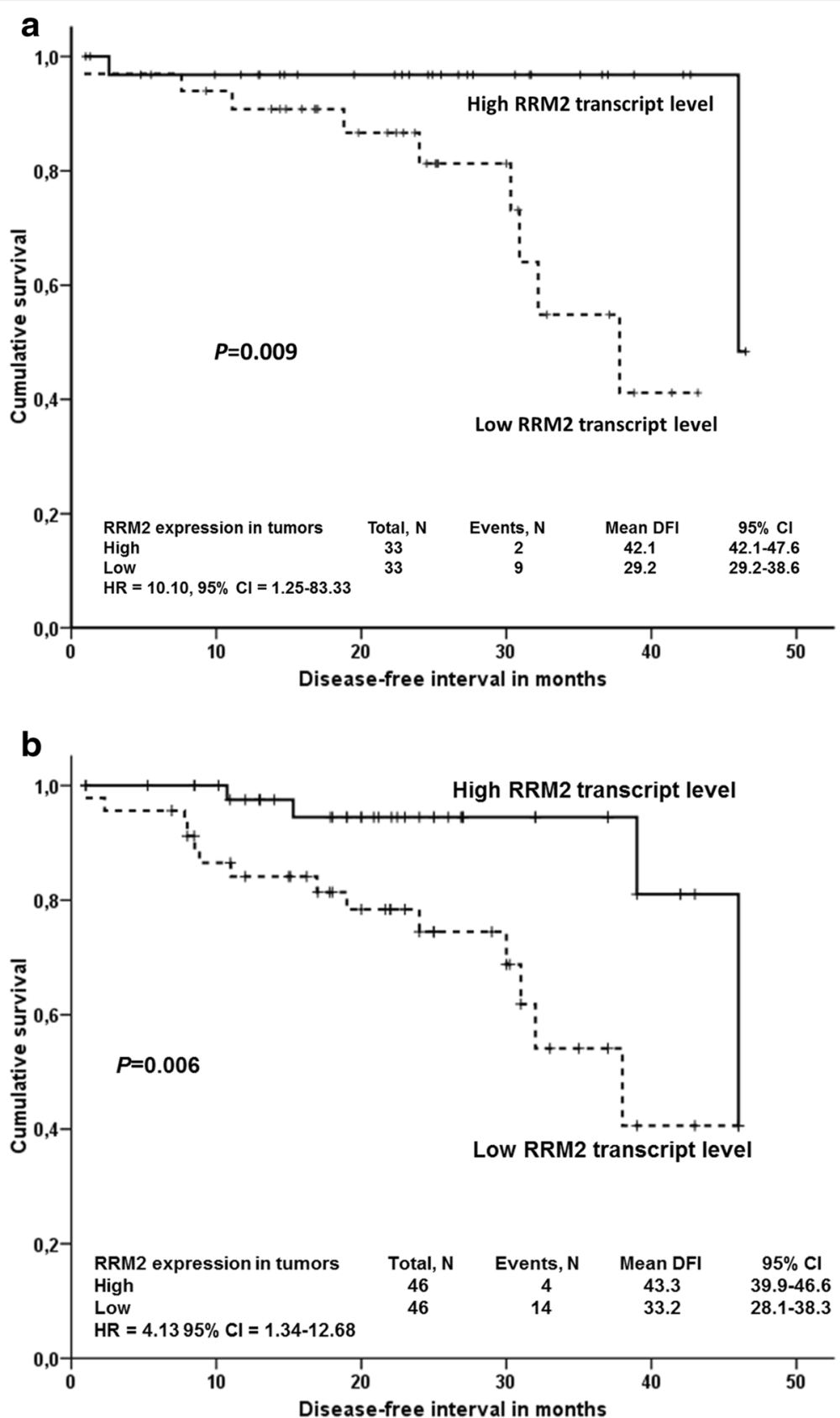

Fig. 2 Association between RRM2 transcript levels and DFI of colorectal cancer patients. Kaplan-Meier survival curves were plotted for patients ( $n=66$, one patient was lost to follow up) from the validation set I (a) or combined testing and validation I sets $(n=92)(\mathbf{b})$. Patients were divided into two groups according to the median of transcript levels in tumors. Dashed line represents the group with lower transcript levels, solid line the group with higher transcript levels than median. Differences between groups were compared using Log-rank test. All genes have been analyzed, but to retain concise style only significant association is reported. $\mathrm{HR}=$ hazard ratio, $95 \% \mathrm{Cl}=95 \%$ confidence intervals for stage-adjusted analyses

Protein levels in tumors and adjacent non-malignant mucosa

DPYS and UPB1 protein levels were analyzed in a subset of the testing set used for the methylation study, enabling an evaluation of the cascade of methylation, gene, and protein expression levels in colorectal cancer samples (Fig. 4). However, DPYS and UPB1 protein levels did not significantly correlate either with their transcripts or methylation levels $(P>0.05)$.

\section{Discussion}

The questions connected with prognostic importance of molecular profile of 5-FU pathway in colorectal cancer remain attractive topics throughout last 15 years. Existing 


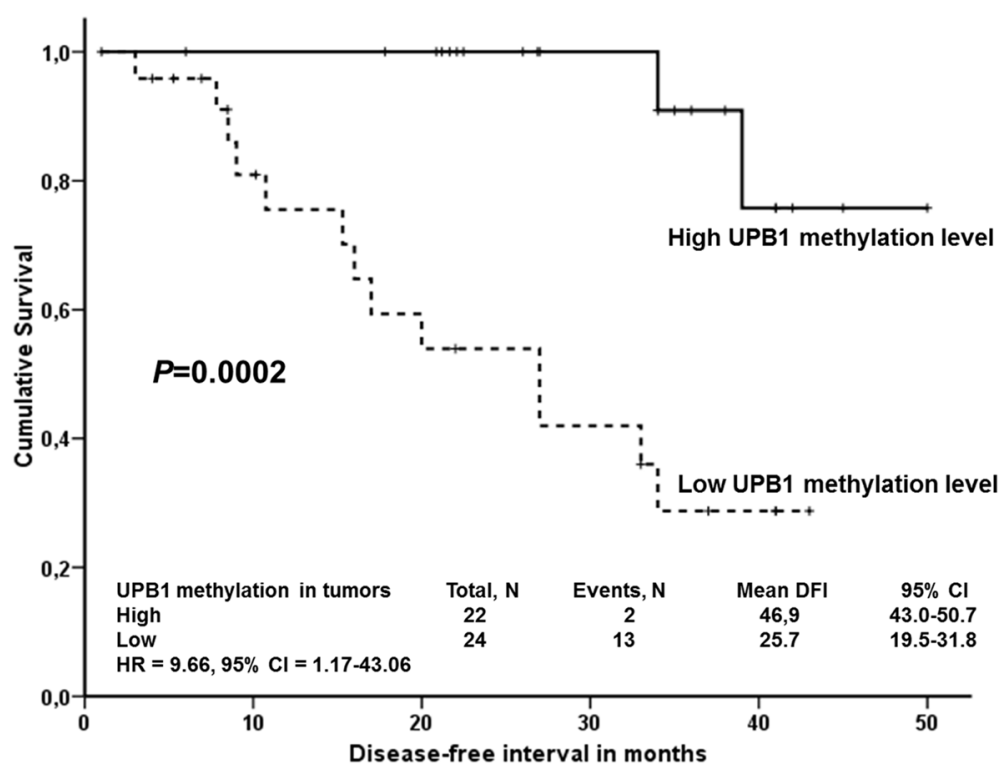

Fig. 3 Association between UPB1 methylation levels and DFI of colorectal cancer patients. Kaplan-Meier survival curves were plotted for patients from the both testing and validation II sets combined $(n=46)$. Seven stage IV patients were excluded and for further 31 patients data on methylation or DFI were not available. Patients were divided into two groups according to the median of intratumoral gene methylation levels. Dashed lines represent the group with lower methylation levels and solid lines represent the group with higher levels than median. Differences between these groups were compared using Log-rank test. HR= hazard ratio, $95 \% \mathrm{Cl}=95 \%$ confidence intervals for stage-adjusted analyses

studies offered a plethora of mostly conflicting results. The absence of complex understanding, focused on mechanisms of action underlying the most promising biomarkers precludes their translation into clinical setting. Apparently, the final prognostic scheme will integrate clinical factors, e.g., stage and grade of the tumor with a cascade of molecular markers involving genetic, epigenetic, and phenotypic factors. The present study brings completely new insight into this area by comprehensive molecular profiling of major 5-FU pathway genes.

The present study shows for the first time that only three (DPYS, UPB1, and UPP2) out of 15 evaluated 5-
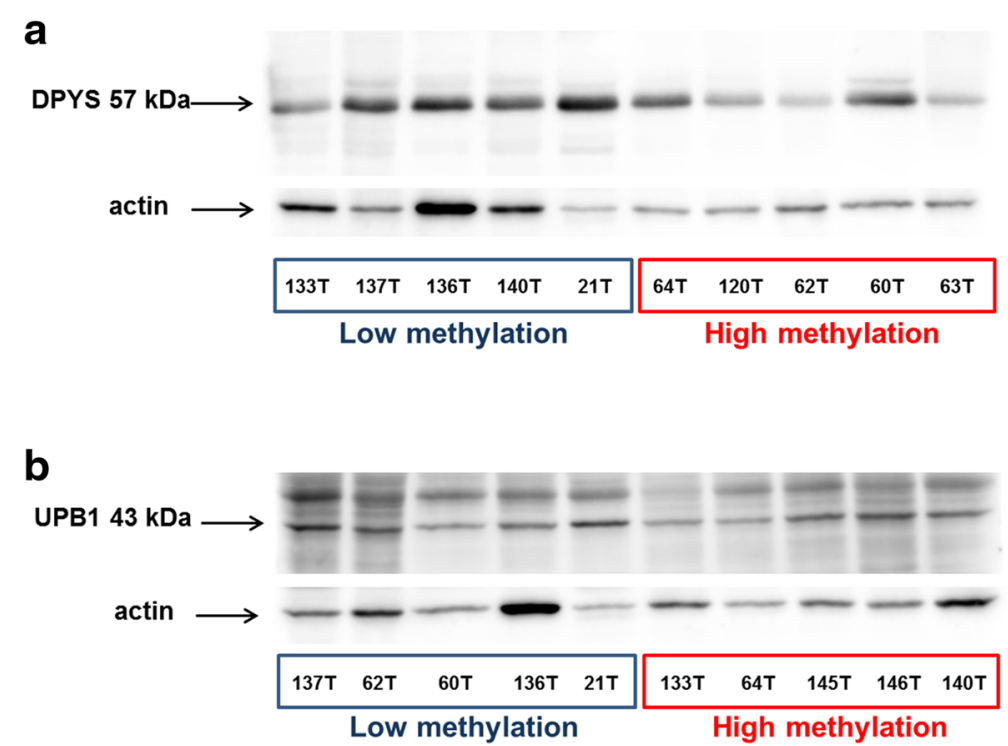

Fig. 4 Protein expression of DPYS and UPB1 in tumors of colorectal cancer patients. Protein expression of DPYS (a) and UPB1 (b) was assessed by immunoblotting with normalization to actin in the representative set of tumors with highest and lowest methylation levels as described in Materials and Methods 
FU genes, are subject to notable methylation in tumor and adjacent mucosa tissues.

Association of UPB1 promoter methylation with worse prognosis of colorectal cancer patients, reported here on two independent groups of patients and in the combined set irrespective of 5-FU treatment, poses a completely novel direction in pharmacogenomics of colorectal cancer. UPB1 is an 5-FU inactivating enzyme [24], responsible for degradation of pyrimidine bases (uracil and thymine) and its genetic defect causes severe forms of propionic acidemia [25]. We hypothesized that a high UPB1 expression in tumor cells caused by promoter demethylation could exert a negative impact on the colorectal cancer patients response to 5-FU. However, we did not prove such association in the combined set of 5-FU treated patients and moreover, UPB1 methylation level did not correlate with either the transcript or the protein levels suggesting that its prognostic role is most probably a complex phenomenon involving some other factors. The lack of such correlation may be explained by a number of effects, e.g., variation in DNA folding in the studied region, regulation of target gene by enhancers/silencers or by other than the followed CpGs or control of gene expression by histone modifications. A more refined screening of $\mathrm{CpG}$ methylation in the $U P B 1$ surrounding area could provide more information about potentially linked epigenetic changes. Moreover, the function of the above mentioned gene may also be modulated by microRNA interference (e.g., hsa-miR-216a, predicted by TargetScan).

From the genetic point of view it is intriguing that recent study reported a strong association between the rs2070474 polymorphism and gastrointestinal toxicity in
5-FU treated cancer patients [26]. It is of interest that this polymorphism lies inside a large CpG island consisting of $98 \mathrm{CpG}$ sites [27] and near to the transcription factor-binding motifs corresponding to a critical regulator of the intestine, the CDX2 (caudal-type homeobox transcription factor 2, OMIM: 600297 [28];). A potential linkage of genetic with epigenetic changes thus should also be considered.

On the basis of our gene expression data we may generalize, that colorectal tumors irrespective of the stage and localization share common downregulation of DPYD and upregulation of PPAT, UMPS, RRM2, and SLC29A1 transcripts. RRM2 and UMPS upregulations and DPYD downregulation in colorectal tumors comply with the previous study [29].

Interestingly, SLC29A1 was recently suggested as potential co-determinant of clinical response to 5-FU [14] and its upregulation demonstrated by the present study further underpins the potential for targeted therapy of colorectal cancer. On the basis of gene expression profile we may deduce that chemotherapy-naïve colorectal cancer patients have in general favorable expression profile shifted towards 5-FU activation (Fig. 5). A potential change of this profile by chemotherapy or during metastatic process presents another interesting question that needs to be addressed.

Moreover, promoter of the 5-FU inactivating enzyme DPYS was found hypermethylated in colorectal tumors by this study. We thus confirmed the previously published DPYS hypermethylation in colon carcinomas (and breast and prostate carcinomas) compared with paired normal tissues from the same patients [30]. Recently, it

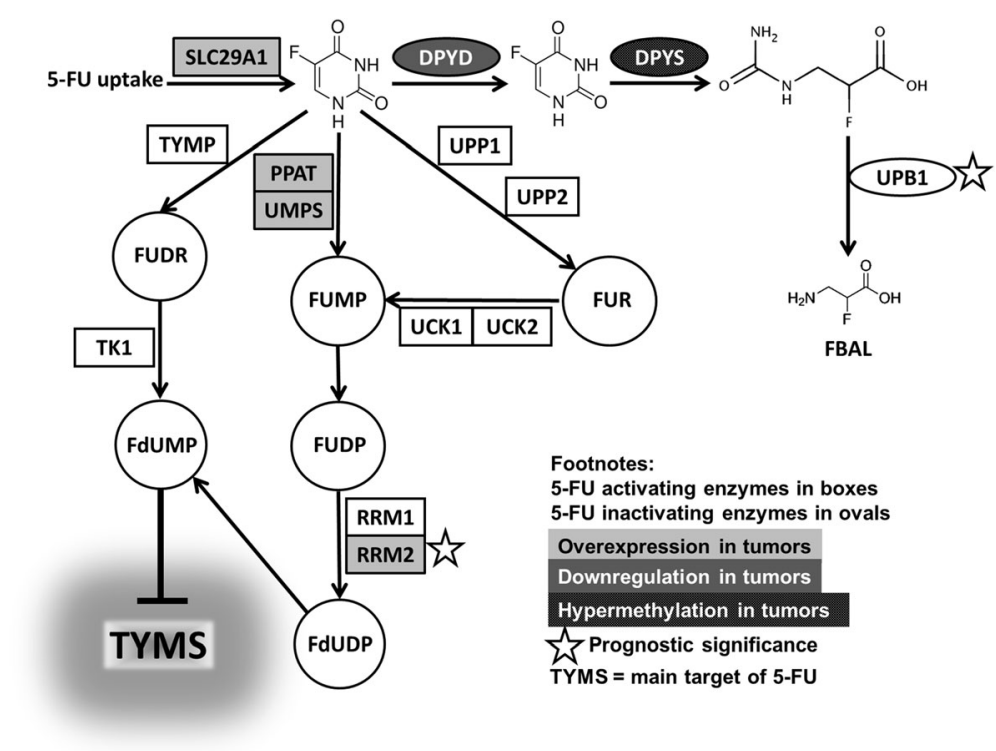

Fig. 5 5-FU pathway genes evaluated by this study (adopted from [24]) 
was reported that differential methylation of $D P Y S$ (and heat shock $27 \mathrm{kDa}$ protein 1, HSPB1, OMIM: 602195 and cyclin D2, CCND2, OMIM: 123833) provides independent prognostic information for prostate carcinoma [31]. Based on the present and earlier studies, colorectal cancer-specific complex prognostic model based on gene expression and methylation profile seems to deserve further exploration.

Prognostic significance of low RRM2 transcript level for poor colorectal cancer patient's outcome observed by the present study contradicts the previously published data. High RRM2 level was poor survival predictor in colorectal cancer patients [32] reflecting the established in vitro ability of RRM2 to enhance cellular invasiveness and genetic instability [33]. We cannot rule out that the qPCR assay for RRM2 employed in the present study also covered the RRM2B (OMIM: 604712) subunit whose protein structure is $80 \%$ identical to RRM2. RRM2B intriguingly exerts opposite activity to RRM2 and its expression associates with a better survival of colorectal cancer patients [34]. On the other hand, RRM2 is 5-FU activating enzyme [24] and thus the result observed by us seems logical from this point of view despite the fact that we have not observed a direct link between prognostic role of RRM2 and 5-FU therapy (perhaps due to the low number of the followed patients). Bearing in mind the issue of study size and publicly available gene expression data, we analyzed the prognostic power of RRM2 expression by SurvExpress [35] tool using data from GSE12945 set $(n=947)$. A borderline significant association towards higher risk of shorter disease-free survival of the patients with lower expression of RRM2 was apparent $(p=0.050$, Additional file 1: Figure S8).

The present study in line with other authors [9], has not confirmed that overexpression of TYMS protein or transcript predicts poor outcome in colorectal cancer patients $[7,8]$. Similarly, the results of studies indicating potential prognostic role of DPYD $[10,11]$ or TYMP [12] expression for survival of colorectal cancer patients after 5-FU-treatment were not replicated.

The small sample size and small patient's groups used for DFI analyses, especially of patients treated with 5-FU pose the major limitations of this study. Nevertheless, we compared the methylation profiles with the publicly available database MethHC (Methylation and gene expression in Human Cancer, http://methhc.mbc.nctu.edu.tw) integrating gene expression, methylation, and microRNA expression data from The Cancer Genome Atlas (TCGA) [36]. Our data complies with the results reported by this database, i.e., the highest levels in UPP2, UPB1, and DPYS (the rest of the genes below $25 \%$ ) and significantly higher methylation of DPYS in tumor compared with mucosa tissues (Additional file 1: Figure S9).
The variability among the patient cohorts could also explain the lack of replication of some results. On the other hand, the use of validation sets helped to achieve more convincing interpretation of the replicated results and where possible the analysis of combined sets increased the study power. The lack of tissue aliquots for simultaneous isolation of RNA and DNA necessitated the use of two different validation sets. This fact precluded us to perform the otherwise preferable combined analyses of both validation sets. Consequently, missing data for comparison of methylation levels with DFI may be seen as a study limitation.

\section{Conclusions}

In this study, we addressed importance of genes involved in the 5-FU pathway for the prognosis of colorectal cancer patients. In conclusion, chemotherapy-naïve colorectal tumors seem to have favorable 5-FU pathway gene expression profile. Additionally, low RRM2 gene expression and UPB1 methylation level represent treatment-independent poor prognostic factors for colorectal carcinoma patients and should be further investigated in relation to other epigenetic regulation pathways (such as microRNAs) and in a complexity with other relevant systems, such as DNA repair.

\section{Additional file}

Additional file 1: Table S1. Lists TaqMan Gene Expression Assays used in the study. Table $\mathbf{S} 2$ shows sequence of primers and PCR conditions used for promoter CpG methylation profiling. Table S3 shows results of stage-adjusted Cox regression of associations between transcript levels and DFI of colorectal cancer patients from the combined testing and validation I sets. Figure $\mathbf{S 1}$ depicts 5-Fluorouracil pathway gene expression levels in the studied sets of colorectal cancer patients. Figure S2 shows results of analysis of associations between transcript levels and disease-free survival of colorectal cancer patients from the validation set I. Figure S3 shows results of analysis of associations between transcript levels and disease-free survival of colorectal cancer patients from the testing set. Figure S4 shows results of analysis of associations between transcript levels and disease-free survival of colorectal cancer patients from the combined testing and validation I set. Figure S5 shows results of analysis of associations between transcript levels and disease-free survival of 5-fluorouracil-treated colorectal cancer patients from the combined testing and validation I set. Figure S6 shows results of analysis of associations between transcript levels and disease-free survival of untreated colorectal cancer patients from the validation I set. Figure S7 shows results of analysis of associations between UPB methylation levels and disease-free survival of colorectal cancer patients. Figure S8 shows analysis of association of RRM2 expression with disease-free survival of colorectal cancer patients based on publicly available GEO database. Figure S9 shows analysis of methylation profiles of 5-FU pathway genes in human colorectal tumor (red boxes) and mucosa (green boxes) tissues from publicly available MethHC database. (DOC $1916 \mathrm{~kb}$ )

\section{Abbreviations}

5-FU: 5-fluorouracil; 95 \% Cl: 95 \% confidence interval; CCND2: Cyclin D2; CDNA: Complementary DNA; CDX2: Caudal-type homeobox transcription factor 2; CpG: Cytosine-phosphate-guanine; CR: Complete response;

DFI: Disease-free interval; DFS: Disease-free survival; DPYD: Dihydropyrimidine 
dehydrogenase; DPYS: Dihydropyrimidinase; dTTP: Deoxythymidine triphosphate; EIF2B1: Eukaryotic translation initiation factor 2B, subunit 1; FDR: False discovery rate; FdUMP: Fluorodeoxyuridine monophosphate; GEO: Gene expression omnibus; HR: Hazard ratio; HRM: High resolution melting; HSPB1: Heat shock 27kDa protein 1; ICD-10: The international classification of diseases, version 10; MIQE: Minimum information for publication of qPCR experiments; MRPL19: Mitochondrial ribosomal protein L19; OMIM: Online mendelian inheritance in man; PCR: Polymerase chain reaction; PD: Progression of the disease; PharmGKB: The pharmacogenomics knowledgebase; POLR2A: DNA-directed RNA polymerase II subunit A; PPAT: Phosphoribosylpyrophosphate amidotransferase; PR: Partial response; PSMC4: Proteasome (prosome, macropain) 26S subunit, ATPase, 4; QPCR: Quantitative real-time PCR; RECIST: Response evaluation criteria in solid tumors; RRM1/2: Ribonuclease reductase subunit M1/2; SD: Stabilization of the disease; SLC29A1: Solute carrier transporter 29A1; Ta: Annealing temperature; TK1: Thymidine kinase; Tm: Melting temperature; TYMP: Thymidine phosphorylase; TYMS: Thymidylate synthase; UCK1/ 2: Uridine cytidine kinase 1/2; UICC: Union for international cancer control; UMPS: Uridine monophosphate synthetase; UPB1: Beta-ureidopropionase; UPP1/2: Uridine phosphorylase 1/2

\section{Acknowledgements}

Not applicable.

\section{Funding}

This work was supported by the Internal Grant Agency of the Czech Ministry of Health (project no.: NT/14329-3 to V.L.), Czech Science Foundation (project no.: P301/12/1585 to P.V.), Grant Agency of Charles University in Prague (project no. 1200314 to T.K.), and by the National Sustainability Program I (NPU I) (project no. LO1503 to P.S.).

\section{Availability of data and materials}

The datasets during and/or analyzed during the current study available from the corresponding author on reasonable request.

\section{Authors' contributions}

TK, PaP, IK, PB, PM, SS, and HV carried out the experimental studies, participated in the evaluation of results, and drafted the manuscript. PS, VL, and PP designed and coordinated the study, performed statistical analyses, and drafted the manuscript. NP, SM, PiP, BJ and VO recruited patients, collected clinical data, and drafted the manuscript. All authors read and approved the final manuscript.

\section{Authors' information}

Not applicable.

\section{Competing interests}

The authors declare that they have no competing interests.

\section{Consent for publication}

Not applicable.

\section{Ethics approval and consent to participate}

All patients, aware of the study aims, methods and potential risks, signed consensually an informed consent form. The study was approved by the Ethical Committees of the Medical Faculty and Teaching Hospital in Pilsen and General Teaching Hospital in Prague, Czech Republic.

\section{Author details}

'Department of Toxicogenomics, National Institute of Public Health, Prague, Czech Republic. ${ }^{2}$ Third Faculty of Medicine, Charles University, Prague, Czech Republic. ${ }^{3}$ Biomedical Centre, Medical School Pilsen, Charles University in Prague, Pilsen, Czech Republic. ${ }^{4}$ Department of Molecular Biology of Cancer, Institute of Experimental Medicine, Czech Academy of Sciences, Videnska 1083, 14200 Prague 4, Czech Republic. ${ }^{5}$ Deparment of Surgery, Teaching Hospital and Medical School Pilsen, Charles University in Prague, Pilsen, Czech Republic. ${ }^{6}$ Department of Surgery, General University Hospital in Prague, First Medical Faculty, Charles University, Prague, Czech Republic. ${ }^{7}$ Toxicogenomics Unit, National Institute of Public Health, Srobarova 48, 100 42 Prague 10, Czech Republic.
Received: 4 January 2016 Accepted: 30 September 2016

Published online: 12 October 2016

\section{References}

1. Bray F, Ren JS, Masuyer E, et al. Global estimates of cancer prevalence for 27 sites in the adult population in 2008. Int J Cancer. 2013;132:1133-45.

2. Jemal A, Bray F, Center MM, et al. Global cancer statistics. CA Cancer J Clin. 2011;61:69-90.

3. Meyerhardt JA, Mayer RJ. Systemic therapy for colorectal cancer. N Engl J Med. 2005;352:476-87.

4. Diasio RB, Harris BE. Clinical pharmacology of 5-fluorouracil. Clin Pharmacokinet. 1989;16:215-37.

5. Mattison LK, Soong R, Diasio RB. Implications of dihydropyrimidine dehydrogenase on 5 -fluorouracil pharmacogenetics and pharmacogenomics. Pharmacogenomics. 2002;3:485-92.

6. Wilson PM, Danenberg PV, Johnston PG, et al. Standing the test of time: targeting thymidylate biosynthesis in cancer therapy. Nat Rev Clin Oncol. 2014:11:282-98.

7. Donada M, Bonin S, Barbazza R, et al. Management of stage II colon cancer - the use of molecular biomarkers for adjuvant therapy decision. BMC Gastroenterol. 2013;13:36.

8. Lu Y, Zhuo C, Cui B, Liu Z, et al. TYMS serves as a prognostic indicator to predict the lymph node metastasis in Chinese patients with colorectal cancer. Clin Biochem. 2013:46:1478-83.

9. Li S, Zhu L, Yao L, et al. Association between ERCC1 and TS mRNA levels and disease free survival in colorectal cancer patients receiving oxaliplatin and fluorouracil (5-FU) adjuvant chemotherapy. BMC Gastroenterol. 2014;14:154.

10. Soong R, Shah N, Salto-Tellez M, et al. Prognostic significance of thymidylate synthase, dihydropyrimidine dehydrogenase and thymidine phosphorylase protein expression in colorectal cancer patients treated with or without 5-fluorouracil-based chemotherapy. Ann Oncol. 2008;19:915-9.

11. Goto T, Shinmura K, Yokomizo K, et al. Expression levels of thymidylate synthase, dihydropyrimidine dehydrogenase, and thymidine phosphorylase in patients with colorectal cancer. Anticancer Res. 2012;32:1757-62.

12. Ogawa M, Watanabe M, Mitsuyama Y, et al. Thymidine phosphorylase mRNA expression may be a predictor of response to post-operative adjuvant chemotherapy with S-1 in patients with stage III colorectal cancer. Oncol Lett. 2014;8:2463-8.

13. Clarke ML, Mackey JR, Baldwin SA, et al. The role of membrane transporters in cellular resistance to anticancer nucleoside drugs. Cancer Treat Res. 2002; 112:27-47.

14. Phua LC, Mal M, Koh PK, et al. Investigating the role of nucleoside transporters in the resistance of colorectal cancer to 5-fluorouracil therapy. Cancer Chemother Pharmacol. 2013;71:817-23.

15. Hlavata I, Mohelnikova-Duchonova B, Vaclavikova R, et al. The role of ABC transporters in progression and clinical outcome of colorectal cancer. Mutagenesis. 2012;27:187-96

16. Slyskova J, Korenkova V, Collins AR, et al. Functional, genetic, and epigenetic aspects of base and nucleotide excision repair in colorectal carcinomas. Clin Cancer Res. 2012;18:5878-87.

17. Therasse P, Arbuck SG, Eisenhauer EA, et al. New guidelines to evaluate the response to treatment in solid tumors. European Organization for Research and Treatment of Cancer, National Cancer Institute of the United States, National Cancer Institute of Canada. J Natl Cancer Inst. 2000;92:205-16.

18. Brynychova V, Hlavac V, Ehrlichova M, et al. Importance of transcript levels of caspase- 2 isoforms $S$ and $L$ for breast carcinoma progression. Future Oncol. 2013;9:427-38.

19. Soucek P, Azenbacher P, Skoumalova I, et al. Expression of cytochrome P450 genes in CD34+ hematopoetic stem and progenitor cells. Stem Cells. 2005:23:1417-22.

20. Hlavac V, Brynychova V, Vaclavíkova R, et al. The role of cytochromes P450 and aldo-keto reductases in prognosis of breast carcinoma patients. Medicine. 2014;93:e2552014.

21. Bustin SA, Benes V, Garson JA, et al. The MIQE guidelines: minimum information for publication of quantitative real-time PCR experiments. Clin Chem. 2009:55:611-22.

22. Hlavac V, Brynychova V, Vaclavikova $R$, et al. The expression profile of ATPbinding cassette transporter genes in breast carcinoma. Pharmacogenomics. 2013;14:515-29. 
23. Pfaffl MW, Horgan GW, Dempfle L. Relative expression software tool (REST) for group-wise comparison and statistical analysis of relative expression results in real-time PCR. Nucleic Acids Res. 2002;30:e36.

24. Thorn CF, Marsh S, Carrillo MW, et al. PharmGKB summary: fluoropyrimidine pathways. Pharmacogenet Genomics. 2011;21:237-42.

25. van Kuilenburg AB, Meinsma R, Beke E, et al. b-Ureidopropionase deficiency: an inborn error of pyrimidine degradation associated with neurological abnormalities. Hum Mol Genet. 2004;13:2793-801.

26. Fidlerova J, Kleiblova P, Kormunda S, et al. Contribution of the $\beta$ ureidopropionase (UPB1) gene alterations to the development of fluoropyrimidine-related toxicity. Pharmacol Rep. 2012;64:1234-42.

27. ENCODE Project Consortium. An integrated encyclopedia of DNA elements in the human genome. Nature. 2012;489:57-74.

28. Verzi MP, Shin $\mathrm{H}$, He HH, et al. Differentiation-specific histone modifications reveal dynamic chromatin interactions and partners for the intestinal transcription factor CDX2. Dev Cell. 2010;19:713-26.

29. Kidd EA, YU J, Li X, et al. Variance in the expression of 5-Fluorouracil pathway genes in colorectal cancer. Clin Cancer Res. 2005;11:2612-9.

30. Chung W, Kwabi-Addo B, Ittmann M, et al. Identification of novel tumor markers in prostate, colon and breast cancer by unbiased methylation profiling. PLoS One. 2008;3:e2079.

31. Vasiljevic N, Ahmad AS, Thorat MA, et al. DNA methylation gene-based models indicating independent poor outcome in prostate cancer. BMC Cancer. 2014;14:655

32. Liu X, Zhang H, Lai L, et al. Ribonucleotide reductase small subunit M2 serves as a prognostic biomarker and predicts poor survival of colorectal cancers. Clin Sci. 2013;124:567-78.

33. D'Angiolella V, Donato V, Forrester FM, et al. Cyclin F-mediated degradation of ribonucleotide reductase $\mathrm{M} 2$ controls genome integrity and DNA repair. Cell. 2012;149:1023-34

34. Liu X, Lai L, Wang X, et al. Ribonucleotide reductase small subunit M2B prognoses better survival in colorectal cancer. Cancer Res. 2011;71:3202-13.

35. Aguirre-Gamboa R, Gomez-Rueda H, Martinez-Ledesma E, et al. SurvExpress: an online biomarker validation tool and database for cancer gene expression data using survival analysis. PLoS One. 2013;8(9):e74250.

36. Huang WY, Hsu SD, Huang HY, et al. MethHC: a database of DNA methylation and gene expression in human cancer. Nucleic Acids Res. 2015;43(Database issue):D856-61.

\section{Submit your next manuscript to BioMed Central and we will help you at every step:}

- We accept pre-submission inquiries

- Our selector tool helps you to find the most relevant journal

- We provide round the clock customer support

- Convenient online submission

- Thorough peer review

- Inclusion in PubMed and all major indexing services

- Maximum visibility for your research

Submit your manuscript at www.biomedcentral.com/submit

) Biomed Central 